\title{
Crustáceos bentónicos y macrófitos como indicadores de calidad ecológica en los lagos de los Andes Australes de Ecuador
}

\author{
Miguel Alonso $^{1,2}$, Pablo Mosquera ${ }^{3}$, Henrieta Hampel ${ }^{4,2}$, Raúl F. Vázquez ${ }^{5,2}$ \\ ${ }^{1}$ Ecology Section. Department of Evolutionary Biology, Ecology and Environmental Sciences, \\ Faculty of Biology, University of Barcelona, Spain. \\ 2 Departamento de Recursos Hídricos y Ciencias Ambientales, Universidad de Cuenca, Ecuador. \\ 3 ETAPA EP, Subgerencia de Gestión Ambiental, Ecuador. \\ ${ }^{4}$ Facultad de Ciencias Químicas, Universidad de Cuenca, Ecuador. \\ 5 Facultad de Ingeniería, Universidad de Cuenca, Ecuador. \\ Autor para correspondencia: malonso@ub.edu \\ Fecha de recepción: 1 de marzo 2017 - Fecha de aceptación: 8 de mayo 2017
}

\section{RESUMEN}

Se ha estudiado la composición taxonómica de las comunidades de crustáceos litoral-bentónicos (branquiópodos, copépodos y ostrácodos) y macrófitos de las masas de agua lacustre del Parque Nacional Cajas situado en los Andes Australes de Ecuador, con objeto de identificar los taxones más adecuados para ser utilizados como indicadores del estado ecológico de los lagos. De los crustáceos (22 taxones) se diferencian tres grupos. Un primer grupo con taxones de alto valor indicador formado por Branchinecta papillata, Ilyocryptus spinosus, Alonella sp, Pleuroxus sp. Paralona pigra y Alona sp. gr. manueli. Un segundo grupo con taxones de valor indicador medio formado por Alona glabra, Alona guttata, Microcyclops sp. Eucyclops sp. y Paracyclops sp. El tercer grupo estaría formado por taxones de escaso valor indicador, bien por su carácter eurioico y/o cosmopolita o por su rareza. De los macrófitos se han valorado como buenos indicadores del estado ecológico y de conservación de los lagos, los taxones enraizados sumergidos y flotantes para la masa de agua, y los helófitos e higrófitos para los litorales.

Palabras clave: Crustáceos, macrófitos, indicadores, calidad ecológica, Andes, Sur de Ecuador.

\begin{abstract}
The taxonomic composition of the litoral benthic limnetic crustacean communities, (branchiopods, copepods and ostracods) and macrophytes of the lacustrine water bodies of the Cajas National Park located in the Southern Andes of Ecuador have been studied in order to identify the most suitable taxa to be used as indicators of the ecological status of lakes. Among the crustaceans (22 taxa) three groups were differentiated. A first group with taxa of higher indicator value including Branchinecta papillata, Ilyocryptus spinosus, Alonella sp, Pleuroxus sp. Paralona pigra and Alona sp. gr. manueli. A second group with medium indicator value including Alona glabra, Alona guttata, Microcyclops sp. Eucyclops sp. and Paracyclops sp. The third group was formed by taxa of the lower indicator value, either because of its eurioic and / or cosmopolitan character or because of its rarity. Macrophytes have been evaluated as good indicators of the ecological and conservation status of the lakes. The taxa which were submerged, floating and rooted in the sediment were identified to be a good indicator for the water body, and helophytes and hygrophytes for the limnetic litoral zone.
\end{abstract}

Keywords: Crustaceans, macrophytes, indicators, ecological quality, Andes, South of Ecuador. 


\section{INTRODUCCION}

Las relaciones encontradas entre la presencia de las especies y las características de sus hábitats llevaron a los primeros limnólogos a establecer sistemas de indicadores biológicos (Margalef, 1955). Éstos han venido siendo posteriormente utilizados para estudios sobre la calidad de los ecosistemas acuáticos, entendiendo que los organismos integran toda una información que es difícil obtener por otros medios. Inicialmente fueron utilizados para el análisis de la calidad del agua de las aguas fluyentes (Helawell, 1978) y, posteriormente, se fueron adaptando a los medios lacustres. En éstos últimos, los invertebrados considerados son principalmente los quidóridos (Branchiopoda, Anomopoda) (De Eyto et al., 2002), los cuales están notablemente diversificados, son cosmopolitas y poseen características autoecológicas muy definidas a nivel de especie. Además, dejan restos reconocibles en el sedimento que permiten llevar a cabo estudios paleolimnológicos destinados a evaluar cambios en el estado trófico o de acidificación de los lagos (Frey, 1960 \& 1988; Harmsworth \& Whiteside, 1968; Whiteside, 1970; Boucherle \& Züllig, 1983; Hofmann, 1987; Hann et al., 1994). Los macrófitos por su parte han sido también ampliamente utilizados como indicadores de eutrofización de los lagos. Se asume que, por un lado, los incrementos de nutrientes, particularmente de $\mathrm{PO}_{4}^{-3}$, pueden provocar un aumento de la densidad y de la biomasa de los macrófitos, alterando los ciclos biogeoquímicos, la calidad del agua, los hábitats de otras comunidades biológicas (peces, invertebrados, aves acuáticas) y los usos de los lagos (Barko et al., 1986; Carpenter \& Lodge, 1986; Alexander et al., 2008). Por otro lado, algunos macrófitos también pueden ser considerados como indicadores de eutrofización, cuando sus poblaciones se encuentran anormalmente reducidas por el crecimiento del fitoplancton, lo cual reduce la transparencia del agua (Chambers \& Kalff, 1985; Kiersch et al., 2004).

En el presente trabajo se analizaron las comunidades de crustáceos bentónicos (branquiópodos, copépodos y ostrácodos) y macrófitos de los lagos, lagunas y charcas del Parque Nacional Cajas (PNC), considerando estas masas de agua como representativas del conjunto lacustre existente en los Andes Australes de Ecuador. El objeto del análisis es asignar niveles de importancia relativa a los diferentes taxones, en cuanto a su potencial uso como indicadores de calidad ecológica de los lagos de los Andes Australes de Ecuador.

\section{MATERIAL Y MÉTODOS}

Los muestreos se llevaron a cabo en 149 (para crustáceos) y 178 (para macrófitos) masas de agua lacustre (lagos, lagunas y charcas) ubicadas entre 3,150 y 4,460 m.s.n.m. en el Parque Nacional Cajas (Andes Australes de Ecuador) a lo largo del año 2015. Este programa de muestreo se realizó en el marco del proyecto "Caracterización Limnológica de los lagos y lagunas del Parque Nacional Cajas", financiado por la Universidad de Cuenca y ETAPA. Las muestras de crustáceos se tomaron en la zona litoral de los lagos y en las charcas, mediante un salabre provisto de un mango largo y equipado con una red cónica de $20 \mathrm{~cm}$ de diámetro y $100 \mu \mathrm{m}$ de abertura de poro. Las muestras se introdujeron en envases de plástico y se conservaron en formaldehido al 4\%. En el laboratorio, los especímenes recolectados se separaron de la muestra bajo un microscopio binocular estereoscópico, se colocaron sobre un portaobjetos (en una gota de una mezcla de glicerol-formaldehído), se diseccionaron y se estudiaron bajo un microscopio óptico. Las muestras de macrófitos se recogieron a mano en el litoral y las zonas vadeables; en las zonas profundas se recogieron desde una embarcación con la ayuda de un rastrillo fijado en el extremo de una cuerda con la longitud necesaria en cada caso para acceder al fondo. Las muestras se conservaron en pliegos de papel absorbente y se trasladaron al laboratorio para su determinación.

Las masas de agua lacustre se clasificaron de acuerdo con sus características morfométricas (superficie, volumen, profundidad media, profundidad relativa y régimen de estratificación y mezcla en: lago profundo monomictico (A), lago profundo polimictico (B), lago somero con área grande (C), lago somero (D) y charca (E). 


\section{RESULTADOS Y DISCUSIÓN}

Las comunidades de crustáceos bentónicos y macrófitos son muy uniformes en todos los tipos de masas de agua identificados en el PNC. Aunque se hayan encontrado diferencias hidromorfológicas muy importantes entre ellos, las características del agua y los ambientes lacustres donde se desarrolla la vida, son similares en todas las masas de agua analizadas.

\subsection{Crustáceos}

En total se han identificado 15 taxones de branquiópodos bentónicos más un número indeterminado de taxones del género Alona que aún no han sido descritos para la ciencia; 4 géneros de copépodos bentónicos, más otros pertenecientes a este grupo del orden Harpacticoida, no determinados; y dentro de los ostrácodos, 3 géneros además de otros taxones no determinados. El número de taxones por muestra fluctuó entre 2 y 12, mientras que el valor medio fue en torno a 4. En la Tabla 1 (ver anexo) se presenta el porcentaje de taxones que aparece en cada tipo de masas de agua.

En todos los tipos de lagos los taxones más frecuentes son Chydorus cf. sphaericus y Alona ossiani. Este patrón se da también en el Reino Paleártico con C. sphaericus y Alona affinis (Alonso, 1998; De Eyto et al., 2002) especies que se comportarían como vicariantes de las de los lagos del PNC, y que son también muy próximas morfológicamente, en concreto la última mencionada (Sinev, 1998). Alona ossiani, como vicariante de A. affinis puede considerarse como una especie pionera, colonizando nuevos hábitats incluso cuando la vegetación macrofítica no se encuentra aún bien establecida, o incluso cuando existen signos de perturbación de los hábitats (Whiteside, 1970; Robertson, 1990; Duigan, 1992). Chydorus cf. sphaericus, por su parte, es conocido por su carácter ubicuo y tolerante a un amplio rango de condiciones (Fryer, 1993), incluso a la eutrofización elevada (Mezquita \& Miracle, 1997). Esto lleva a pensar que estas especies, si bien son muy características en el PNC, su valor indicador de posibles cambios ambientales es relativo.

Otros crustáceos que aparecen en todos los tipos de lagos, aunque con menor frecuencia, son los branquiopodos Daphnia pulex y Alonella sp; y todos los copépodos ciclopoides y los harpacticoides. Daphnia pulex es una especie que se encuentra tanto en el plancton como en el litoral de los grandes lagos, y también en las masas de agua de pequeño volumen (Alonso, 1998). Alonella sp., por su parte, es un género muy característico de los bentos lacustres, donde aparece entre la vegetación acuática; este género ha dado para el PNC una especie que actualmente se está describiendo como nueva para la ciencia. Todas las especies del género Alona no determinadas (Alona sp. pl.) también son bentónicas. De los cuatro géneros de ciclopoides inventariados, sólo Metacyclops sp. puede aparecer tanto en el plancton como en el litoral de los lagos. Los otros tres (Microcyclops, Eucyclops y Paracyclops) son estrictamente bentónicos (Kiefer, 1978). Los harpacticoides, son todos bentónicos. El valor de estos crustáceos como indicadores de la calidad ecológica de los lagos del PNC es más elevado que en el caso anterior. Aunque no se observan diferencias significativas de su abundancia entre los diferentes tipos de lagos, sí podrían considerarse componentes muy característicos de la comunidad propia de las masas de agua lacustre del PNC que, como se ha comentado, es muy uniforme. En particular, si Alonella sp. ha podido evolucionar genéticamente en estos ambientes, constituye un indicador ecológico ideal. Cambios observados en la composición de esta comunidad podrían ser interpretados como posibles alteraciones de la calidad ecológica de los lagos.

Un tercer grupo lo forman taxones que no aparecen en todos los tipos de lagos. Todos son también característicos de los bentos litorales lacustres y sólo discriminan los lagos propiamente dichos de las charcas. Estos son los branquiópodos Ceriodaphnia dubia, Ilyocryptus spinosus, Pleuroxus sp, Paralona pigra, Alona guttata y Alona glabra, y los ostrácodos no determinados. Paralona pigra e I. spinosus son las especies con exigencias ambientales más claras. Paralona pigra muestra una clara preferencia por aguas poco mineralizadas y ácidas (De Eyto, 2002). Ilyocryptus spinosus es propia de ambientes lacustres litorales y profundos, con limos orgánicos de granulometría muy fina (Kotov \& Stifter, 2006). La autoecología de A. glabra no está muy definida, ya que esta especie ha aparecido en zonas de salar (De los Rios \& Kotov, 2015), por lo que sería recomendable un análisis taxonómico detallado ya que en ella se han distinguido además dos diferentes filogrupos (Elías-Gutiérrez et al., 2008); al menos en el PNC se comporta como característica de aguas muy poco mineralizadas. Alona guttata es una especie 
cosmopolita y bastante eurioica (Alonso, 1996) por lo que su valor indicador en el PNC es relativo. Pleuroxus sp. es, aparentemente, una especie aún no descrita científicamente; si se demostrase que en efecto es una nueva especie, estaría en el mismo caso que Alonella sp. y constituiría un buen indicador ecológico para el PNC.

Un cuarto grupo lo forman crustáceos poco frecuentes o raros. Estos serían Branchinecta papillata, Simocephalus vetulus, Macrothrix cf. hirsuticornis, Alona sp. gr. manueli, Camptocercus dadayi, Cypridopsis sp. Herpetocypris sp. y Candona sp. El anostráceo B. papillata es exclusivo de lagos someros y charcas temporales, y en el caso de las poblaciones del PNC, de aguas muy poco mineralizadas, lo que aconseja una revisión taxonómica de esta especie ya que su descripción original se basó en ejemplares recogidos en aguas muy mineralizadas (Rogers et al., 2008). Simocephalus vetulus, que es una especie muy común en lagunas y en el litoral de grandes lagos (Alonso, 1996), en el PNC es muy poco frecuente, y lo mismo sucede con M. cf. hirsuticornis y los ostrácodos Cypridopsis sp. Herpetocypris sp. y Candona sp. Alona sp. gr. manueli es otro taxón que se está describiendo como nuevo para la ciencia, característico de aguas poco mineralizadas en localidades de alta montaña y que en el PNC se muestra como propio de lagos someros. El género Camptocercus suele formar poblaciones poco numerosas (Smirnov, 1996), por lo que suele ser considerado como poco frecuente; en el PNC $C$. dadayi aparece sólo en el litoral de lagos someros. El valor de estos taxones como indicadores ecológicos para las masas de agua lacustre del PNC sería muy elevado en el caso de B. papillata por sus requerimientos tan específicos, y de $A$. sp. gr. manueli por tratarse de un taxón que se ha diferenciado genéticamente en este ámbito territorial. El valor indicador del resto de los taxones considerados en este apartado de taxones poco frecuentes o raros, se considera escaso.

\subsection{Macrófitos}

Se han identificado 30 taxones de macrófitos (ver anexo Tabla 2). Diez son macrófitos enraizados sumergidos; 11 higrófitos del litoral; 7 helófitos, que están enraizados en el vaso lacustre, pero tienen la parte vegetativa aérea; 2 macrófitos enraizados flotantes; y un macrófito flotante. Los macrófitos enraizados sumergidos y flotantes están considerados como los mejores indicadores del estado ecológico de los lagos ya que dependen del desarrollo del fitoplancton (estado trófico) que reduce la transparencia del agua (Chambers \& Kalff, 1985). Pero en los lagos del PNC el estado trófico así entendido es muy bajo, fundamentalmente por escasez de nutrientes. Además, el color y la turbidez asociados a la presencia de carbono orgánico particulado (POC) y carbono orgánico disuelto (DOC), procedente de los Andosoles, el bosque y el pajonal de sus cuencas vertientes, puede limitar la luz necesaria para el crecimiento de los macrófitos enraizados sumergidos. Como el POC y el DOC presentes en los lagos debe considerarse de origen natural en las masas de agua lacustre del PNC, hay que considerar que los macrófitos enraizados sumergidos presentes en ellas han alcanzado en la actualidad su máximo desarrollo, de forma que el seguimiento del estado ecológico de los lagos, utilizando estos organismos, debe basarse en que se mantenga la composición específica encontrada en la actualidad y que los hábitats potencialmente adecuados para cada especie, en función de la penetración de la luz y de la naturaleza del sustrato, presenten el máximo nivel de ocupación.

Los helófitos e higrófitos son también de interés como indicadores del estado de conservación de las comunidades litorales, que también controlan la productividad de los lagos y son las que mayor atención reciben de los usuarios de los lagos y constituyen el hábitat de muchas especies de vertebrados (Clayton \& Edwards, 2006). En las masas de agua lacustre del PNC, las comunidades litorales de macrófitos se mantienen sin alteraciones significativas, salvo en algunos puntos por donde accede el ganado a los lagos. De esta forma, todas las especies inventariadas pueden utilizarse como indicadoras, aunque teniendo en cuenta también la estructura de las comunidades, cuya situación actual puede considerarse como de referencia. 


\section{CONCLUSIONES}

En breve, las siguientes son las conclusiones principales derivadas del desarrollo de la presente investigación:

- Las masas de agua lacustre del PNC constituyen un ejemplo representativo del conjunto de lagos de los Andes Australes de Ecuador, lo cual permite, con cierto margen de confianza, extrapolar los resultados del presente estudio a un ámbito territorial más amplio.

- La composición específica de las comunidades de crustáceos y macrófitos es muy uniforme en todos los tipos de masas de agua lacustre del PNC, sólo la de las charcas posee como presencia exclusiva la del anostráceo Branchinecta papillata.

- Prácticamente todas las masas de agua lacustre prospectadas se encuentran sin alteraciones significativas de origen antrópico, lo que permite considerarlas como de referencia para posteriores estudios sobre su calidad ecológica.

- Dentro de los crustáceos se pueden establecer tres grupos de taxones de acuerdo con su posible valor indicador basado en su fidelidad a las masas de agua lacustre del PNC: (1) taxones con valor indicador más alto: Branchinecta papillata, Ilyocryptus spinosus, Alonella sp, Pleuroxus sp. Paralona pigra y Alona sp. gr. manueli; (2) taxones con valor indicador medio: Alona glabra, Alona guttata, Microcyclops sp. Eucyclops sp, Paracyclops sp. y cualquier especie de las denominadas como Alona sp. Pl; (3) el resto de taxones identificados tendrían un valor relativamente bajo en el contexto analizado en el presente trabajo.

- No se han podido asociar valores indicadores de estado ecológico a las especies de macrófitos. Como tipo biológico, el de los macrófitos enraizados sumergidos y flotantes sería el de mayor valor indicador para evaluar el estado de las masas de agua propiamente dichas. Por su parte, los helófitos y los higrófitos serían los de mayor valor indicador para evaluar el estado de los litorales.

\section{AGRADECIMIENTOS}

El presente trabajo se ha llevado a cabo en el ámbito del proyecto "Caracterización limnológica de los lagos y lagunas del Parque Nacional Cajas", patrocinado por la Subgerencia de Gestión Ambiental de ETAPA EP y la Dirección de Investigación de la Universidad de Cuenca (DIUC) y dirigido por la tercera autora. La preparación del presente artículo ha sido factible gracias a becas otorgadas al primer autor y a la tercera autora por parte de la Secretaría de Educación Superior, Ciencia, Tecnología e Innovación del Ecuador (SENESCYT) a través de su Programa "PROMETEO Viejos Sabios".

\section{BIBLIOGRAFIA}

Alexander ML, Woodford MP, Hotchkiss SC (2008). Freshwater macrophyte communities in lakes of variable landscape position and development in northern Wisconsin, U.S.A. Aquatic Botany 88:77-86

Alonso M (1996). Crustacea, Branchiopoda. En: Fauna Iberica, Vol 7. Ramos et al. (Eds.). Madrid, España: Museo Nacional de Ciencias Naturales. CSIC, 486 pp

Alonso M (1998). Las lagunas de la España peninsular. Limnetica 15:1-176

Barko JW, Adams MS, Clesceri NL (1986). Environmental factors and their consideration in the management of submersed aquatic vegetation: a review. Journal Aquatic Plant Management 24:1-10 
Boucherle MM, Züllig H (1983). Cladoceran remains as evidence of change in trophic state in three Swiss lakes. Hydrobiologia 103:141-146

Carpenter SR, Lodge DM (1986). Effects of submersed macrophytes on ecosystem processes. Aquatic Botany 26:341-370

Chambers PA, Kalff J (1985). Depth distribution and biomass of submersed aquatic macrophyte communities in relation to Secchi depth. Canadian Journal of Fisheries and Aquatic Sciences 42:701-709

Clayton J, Edwards T (2006). Aquatic plants as environmental indicators of ecological condition in New Zealand lakes. Hydrobiologia 570:147-151

De Eyto E, Irvine K, Free G (2002). The use of members of the family Chydoridae (Anomopoda, Branchiopoda) as an indicator of lake Ecological Quality in Ireland. Biology and Environment: Proceedings of the Royal Irish Academy 102B:81-91

De los Rios Escalante P, Kotov A (2015). Checklist of Branchiopoda (Anostraca and Cladocera) of Chilean continental waters. Zootaxa 4027(3):366-388

Duigan C (1992). The ecology and distribution of the litoral freshwater Chydoridae Branchiopoda Anomopoda of Ireland, with taxonomic comments on some species. Hydrobiologia 241:1-70

Elías-Gutiérrez M, Martínez-Jerónimo F, Ivanova NV, Valdez Moreno M, Hebert PDN (2008). DNA barcodes for Cladocera and Copepoda from Mexico and Guatemala, highlights and new discoveries. Zootaxa 1839:1-42

Frey DG (1960). The ecological significance of cladoceran remains in lake sediments. Ecology 41:584-689

Frey DG (1988). Littoral and offshore communities of diatoms, cladocerans and dipterous larvae, and their interpretation in paleolimnology. Journal of Paleolimnology 1:179-191

Fryer G (1993). The freshwater Crustacea of Yorkshire: a faunistic and ecological survey. Leeds, UK: Yorkshire Naturalists' Union and Leeds Philosophical and Literary Society, 312 pp

Hann BJ, Leavitt PR, Chang PSS (1994). Cladocera community response to experimental eutrophication in Lake 227 as recorded in laminated sediments. Canadian Journal of Fisheries and Aquatic Sciences 51:2312-2321

Harmsworth R, Whiteside M (1968). Relation of cladoceran remains in lake sediments to primary productivity of lakes. Ecology 49:998-1000

Helawell JM (1978). Biological surveillance of rivers. Stevenage, England: Water Research Centre, $325 \mathrm{pp}$

Hofmann W (1987). Cladocera in space and time: analysis of lake sediments. Hydrobiologia $145: 315-321$

Kiefer F (1978). Freilebende Copepoda. In: Das zooplankton der binnengewässer, 2. Teil. Stuttgart, Germany: Die Binnengewässer 26.2 Teil, 343 pp

Kiersch B, Mühleck R, Gunkel G (2004) Las macrófitas de algunos lagos alto-andinos del Ecuador y su bajo potencial como bioindicadores de eutrofización. Revista de biologia tropical 52:829-837

Kotov AA, Stifter P (2006). Family Ilyocryptidae (Branchiopoda: Cladocera: Anomopoda). Leiden, the Netherlands: Backuys Publishers, $171 \mathrm{pp}$

Margalef R (1955). Los organismos indicadores en la limnologia. Biologia de las aguas continentales,12. Madrid, España: Inst. For. Inv. Exp., Ministerio Agricultura, 300 pp

Mezquita F, Miracle MR (1997). Chydorid assemblages in the sedimentary sequence of Lake La Cruz, Spain, subject to water level changes. Hydrobiologia 360:277-285

Robertson AL (1990). The population dynamics of Chydoridae and Macrothricidae (Cladocera, Crustacea) form the River Thames, UK. Freshwater biology 24:375-389

Rogers DC, De los Rios P, Zuñiga O (2008), The Anostraca of Chile (Branchiopoda). Journal of Crustacean Biology 28:543-550 
Sinev AY (1998). Alona ossiani sp. n., a new species of the Alona affinis complex from Brazil, deriving from the collection of G.O. Sars (Anomopoda: Chydoridae). Arthropoda Selecta $7(2): 103-110$

Smirnov NN (1996). Cladocera: the Chydorinae and Sayciinae (Chydoridae) of the world. Guides to the identification of the microivertebrates of the Continental Waters of the World, Vol. 11. Amsterdam, the Netherlands: SPB Academic Publishing, 197 pp

Whiteside MC (1970). Danish chydorid Cladocera: modern ecology and core studies. Ecological Monographs 40:79-118

\section{ANEXO}

Tabla 1. Crustáceos bentónicos identificados en los diferentes tipos de masas de agua lacustre del Parque Nacional Cajas, y porcentaje de aparición de cada especie en cada tipo: (A) lago profundo monomictico, (B) lago profundo polimictico, (C) lago somero con área grande, (D) lago somero, y (E) charca.

\begin{tabular}{|c|c|c|c|c|}
\hline Tipos de masas de agua & A-B & $\mathrm{C}$ & $\mathrm{D}$ & $\mathrm{E}$ \\
\hline $\mathrm{N}^{\mathrm{o}}$ de masas de agua lacustre por tipo & 67 & 20 & 40 & 22 \\
\hline \multicolumn{5}{|l|}{ BRANCHIOPODA } \\
\hline Branchinecta cf. papillata (Rogers et al., 2008) & & & 3 & 18 \\
\hline Daphnia pulex (Leydig, 1860) & 7 & 20 & 5 & 14 \\
\hline Simocephalus vetulus (Müller, 1776) & 1 & & & \\
\hline Ceriodaphnia dubia (Richard, 1894) & 3 & 15 & 8 & \\
\hline Macrothrix cf. hirsuticornis (Norman \& Brady, 1867) & 1 & 0 & 8 & \\
\hline Ilyocryptus spinosus (Stifter, 1988) & 1 & 5 & 3 & \\
\hline Alonella sp. & 6 & 10 & 18 & 5 \\
\hline Pleuroxus sp. & 10 & 5 & 8 & \\
\hline Chydorus cf. sphaericus (Müller, 1776) & 84 & 90 & 80 & 64 \\
\hline Paralona pigra (Sars, 1862) & 27 & 25 & 3 & \\
\hline Alona guttata (Sars, 1862) & 22 & 15 & 5 & \\
\hline Alona ossiani (Sinev, 1998) & 63 & 40 & 33 & 45 \\
\hline Alona sp. gr. manueli (Sinev \& Zawisza, 2013) & & & 15 & \\
\hline Alona glabra (Sars, 1901) & 4 & 10 & 5 & \\
\hline Alona sp. pl. & 19 & 15 & 8 & 36 \\
\hline Camptocercus dadayi (Stingelin, 1913) & & & 3 & \\
\hline \multicolumn{5}{|l|}{ COPEPODA } \\
\hline Microcyclops sp. & 21 & 30 & 10 & 14 \\
\hline Metacyclops sp. & 13 & 20 & 25 & 23 \\
\hline Eucyclops sp. & 18 & 5 & 20 & 18 \\
\hline Paracyclops sp. & 9 & 15 & 18 & 18 \\
\hline Harpacticoida sp. pl. & 61 & 60 & 45 & 59 \\
\hline \multicolumn{5}{|l|}{ OSTRACODA } \\
\hline Cypridopsis sp. & 3 & & 3 & \\
\hline Herpetocypris sp. & 1 & & & \\
\hline Candona sp. & & 5 & 3 & \\
\hline Ostracoda sp. pl. & 21 & 10 & 20 & \\
\hline
\end{tabular}


Tabla 2. Relación de taxones de macrófitos identificados en las masas de agua lacustre del Parque Nacional Cajas (MES: macrófitos enraizados sumergidos; HEL: helófitos; HIG: higrófitos; MFL: macrófitos flotantes; MEF: macrófitos enraizados flotantes).

\begin{tabular}{|c|c|c|}
\hline Familia & Especies & Tipos biológicos \\
\hline Characeae & Nitella sp. & MES \\
\hline \multirow[t]{3}{*}{ Isoetaceae } & Isoetes andina Spruce ex Hook. & MES \\
\hline & Isoetes lechleri Mett. & MES \\
\hline & Isoetes killipii C.V. Morton. & MES \\
\hline \multirow[t]{6}{*}{ Cyperaceae } & Carex acutata Boott. & HEL \\
\hline & C. lemanniana Boott. & HEL \\
\hline & C. azuayae Steyerm. & HEL \\
\hline & C. acutata Boott. & HEL \\
\hline & C. toreadora Steyerm. & HEL \\
\hline & Isolepis inundata $\mathrm{R} . \mathrm{Br}$. & HEL \\
\hline \multirow[t]{2}{*}{ Poaceae } & Calamagrostis intermedia (J. Presl) Steud. & HIG \\
\hline & C. planifolia (Kunth) Trin. ex Steud. & HIG \\
\hline \multirow[t]{3}{*}{ Juncaceae } & Juncus ecuadoriensis Balslev. & HIG \\
\hline & J. stipulatus Nees ex Meyen. & HIG \\
\hline & J. arcticus Willd. & $\mathrm{HIG}$ \\
\hline Juncaginaceae & Lilaea scilloides (Poir.) Hauman. & HIG \\
\hline \multirow[t]{3}{*}{ Potamogetonaceae } & P. paramoanus R.R. Haynes \& Holm-Niels. & MES \\
\hline & P. illinoensis Morong. & MES \\
\hline & P. filiformis Pers. & MES \\
\hline Iridaceae & Sisyrinchium palustre Diels. & HIG \\
\hline \multirow[t]{2}{*}{ Plantaginaceae } & Callitriche heteropoda Engelm. ex Hegelm. & MES \\
\hline & Callitriche heterophylla Pursh. & MES \\
\hline Brassicaceae & Cardamine bonariensis Pers. & HIG \\
\hline Asteraceae & Cotula mexicana (DC.) Cabrera. & HIG \\
\hline Crassulaceae & Crassula venezuelensis (Steyerm.) Bywater \& Wickens. & HIG \\
\hline Elatinaceae & Elatine ecuadoriensis Molau. & MFL \\
\hline Lamiaceae & Mentha pulegium $\mathrm{L}$. & HIG \\
\hline Haloragaceae & Myriophyllum quitense Kunth. & MES \\
\hline Polygonaceae & Polygonum hydropiperoides Michx. & HEL \\
\hline \multirow[t]{2}{*}{ Ranunculaceae } & Ranunculus flagelliformis $\mathrm{Sm}$. & MEF \\
\hline & Ranunculus limoselloides Turcz. & MEF \\
\hline
\end{tabular}

